Revue d'histoire de l'Amérique française

AN REVUE D.HISTOIRE DE L'AMÉRIQUE FRANÇAISE

\title{
Les hommes politiques du Québec 1867-1967
}

\section{Robert Boily}

Volume 21, numéro 3a, 1967

Cent ans d'histoire 1867-1967

URI : https://id.erudit.org/iderudit/302714ar

DOI : https://doi.org/10.7202/302714ar

Aller au sommaire du numéro

Éditeur(s)

Institut d'histoire de l'Amérique française

ISSN

0035-2357 (imprimé)

1492-1383 (numérique)

Découvrir la revue

Citer cet article

Boily, R. (1967). Les hommes politiques du Québec 1867-1967. Revue d'histoire de l'Amérique française, 21(3a), 597-634. https://doi.org/10.7202/302714ar d'utilisation que vous pouvez consulter en ligne.

https://apropos.erudit.org/fr/usagers/politique-dutilisation/ 
Robert Boily

LES HOMMES

POLITIQUES

DU QUEBEC

$1867-1967$ 


\section{Robert Boily}

Etudes au Collège Sainte-Croix.

Baccalauréat ès arts, Université de Montréal, 1955.

Licence en droit, Universiłé de Montréal, 1958.

Diplôme de l'Institut d'Etudes politiques, Paris, 1961.

Diplôme d'Etudes supérieures (scolarité de Doctorat), Section Droit constitutionnel et science politique, Faculté de Droit, Paris, 1962.

Professeur au Département de Science politique de l'Université de Montréal depuis 1962.

Enseignement: Systèmes politiques comparés (France, GrandeBretagne, URSS, notamment)

Dirige un séminaire sur les institutions et la vie

Recherches : politique au Québec depuis 1965.

Diverses études portant sur le personnel politique de la province de Québec de 1867 à aujourd'hui, tant au niveau fédéral, municipal, provincial.

Etudes de sociologie électorale, portant sur les origines de Montréal. 


\section{LES HOMMES POLITIQUES DU QUÉBEC $1867 \cdot 1967$}

La loi impériale qui fixait au 1er juillet 1867 l'entrée en vigueur de la nouvelle constitution donnait à l'ancienne colonie française à l'intérieur d'un système fédéral, il est vrai assez flou dans l'esprit de plusieurs, son propre système politique. Par cette loi, votée à Londres dans l'indifférence, cette société, autrefois canadienne mais qui pour conserver son identité deviendra canadienne-française, balottée depuis 1760 d'un régime constitutionnel à un autre, entrait en possession d'un instrument politique important. Instrument politique sur lequel elle pouvait exercer un pouvoir souverain ${ }^{1}$ et qui lui permettait ainsi, théoriquement tout au moins, d'orienter son développement, notamment en matière d'éducation et aussi en matière sociale et économique. Plusieurs, par suite même de ce caractère, virent dans l'Acte de 1867, un sommet, celui qu'atteignaient enfin les hommes politiques canadiens-français à la suite d'une longue lutte constitutionnelle et politique commencée aux lendemains de la conquête anglaise et poursuivie tout au long des années et des générations. Quels que soient les jugements que nous puissions porter sur les textes de 1867 il reste que pour la première fois depuis la conquête la collectivité canadienne-française avait la possibilité d'occuper majoritairement un pouvoir politique souverain.

A côté de cette zone de pouvoir qui leur était concédée les hommes politiques du Québec avaient accès à un autre niveau de gouvernement, celui du fédéral. Toutefois, à ce niveau ils demeuraient dans une situation beaucoup plus près de celle qui avait été la leur jusqu'en 1867: ils étaient à Ottawa une minorité

1 Il faut noter ici que les prouvoirs, que détenait le lieutenant-gouverneur au nom du gouvernement central sur le domaine législatif réservé de la province, tomberont en désuétude, pour le Québec, après Mercier. 
qui pouvait être une minorité "influante" mais d'aucune manière une minorité gouvernante ou dirigeante autrement que par participation.

Sur le plan institutionnel, l'homme politique canadien-français avait done un statut différent selon qu'il participait au pouvoir gouvernemental fédéral ou au pouvoir gouvernemental provincial. Les décisions à prendre à l'un ou l'autre de ces deux ordres de gouvernement pouvaient également affecter le développement du Québec et celui de la société canadienne-française. Aux deux niveaux, la loi de majorité, règle fondamentale de la démocratie parlementaire, jouait. A Ottawa elle faisait des hommes politiques canadiens-français une minorité non seulement ethnique mais politique, ayant accès, il est vrai, par suite de l'importance numérique et économique du Québec, au centre décisionnel, le cabinet, mais beaucoup plus comme un groupe de pression ethnique qu'en véritable détenteur d'un pouvoir de décision. L'expansion de la fédération canadienne par l'addition d'autres provinces et la modification du rapport démographique entre les groupes anglais et français par suite d'une immigration massive d'éléments anglo-saxons, ne firent qu'accentuer ce statut de minorité "influante".2 A Québec, cette loi de majorité permettait, pour la première fois depuis l'introduction du système parlementaire dans l'ancienne colonie française, aux hommes politiques de ce peuple d'occuper en force, ou du moins en situation majoritaire, un pouvoir susceptible de conditionner leur vie et leur avenir d'une manière fondamentale.

Ces hommes qui deviennent ainsi les acteurs d'un nouveau jeu politique dont ils ne pourront pas toujours, il s'en faut de beaucoup, imposer les règles, qui sont-ils et qui furent leurs successeurs jusqu'à nos jours? Si on commence et de plus en plus à parler d'eux, à mieux saisir leurs intérêts et leurs caractères ${ }^{3}$,

2 Par "influant" nous entendons ici un pouvoir latent mais pas nécessairement effectif.

3 Fernand Dumont, "La représentation idéologique des classes au Canada français", Recherches sociographiques, VI, I (janvier-avril 1965) : 9-22. Fernand Ouellet, Histoire économique et sociale du Québec 17601850 (Fides, Montréal et Paris, 1966), 570-596. 
à évaluer leur responsabilité ${ }^{4}$ et leur rôle ${ }^{5}$ on ne les connaît encore que très peu. Ou bien nous possédons sur eux toute une série de renseignements épars comme dans Rumilly ${ }^{6}$ ou encore dans Mason Wade ${ }^{7}$ ou bien des essais de synthèse cherchant à les caractériser, à en déterminer les divers types, leur moment d'apparition et de disparition, comme dans l'excellente étude de Jean-Charles Falardeau "Dles élites traditionnelles aux élites nouvelles" 8, sans parler des rares mémoires ou autobiographies ${ }^{\circ}$. Mais il nous manque encore une étude systématique du personnel politique canadien-français, tout particulièrement une étude de leur origine sociale, qui puisse permettre de confirmer certaines hypothèses et pousser plus loin la connaissance de cette société. C'est cette lacune que nous voudrions commencer à combler par cet article en transmettant quelques-uns des résultats auxquels nous ont conduit nos propres recherches sur le personnel politique québécois ${ }^{10}$.

"Pierre Elliott Trudeau, "Some Obstacles to Democracy in Quebec", dans La Dualité canadienne (ouvrage collectif réalisé par Mason Wade) (U.T.P. et P.U.L. 1960), 241-259.

5 Hubert Guindon, "The Social Evolution of Quebec Reconsidered" dans Marcel Rioux et Yves Martin, French Canadian Society, volume i (Carleton Library No. 18, 1964) : 137-168.

6 Robert Rumilly, Histoire de la Province de Québec (tomes I à XVI, Editions Valiquette; tomes XVII à XXIII, Montréal-Editions; tomes XXIV-XXVI, Editions Chanteclerc; tomes XXVII à XXXIV, Editions Fides).

${ }^{7}$ Mason Wade, Les Canadiens français de 1760 à nos jours (2 vol., LA Cercle du livre de France, 2ème édition, 1966).

8 Jean-Charles Falardeau, "Des élites traditionnelles aux élites nouvelles", dans Le pouvoir dans la société canadienne-française, publié sous la direction de Fernand Dumont et Jean-Paul Montminy (P.U.L., Québec, 1966).

9 Pour fins d'exemples: P.-A. Choquette, Un demi-siècle de vie politique (Beauchemin, 1936), $352 \mathrm{pp}$. - Armand LaVergne, Trente ans de vie nationale (Les Editions du Zodiaque, Montréal, 1934), 228 pp. - Antonio Barrette, Mémoires (Beauchemin, Montréal, 1966), $448 \mathrm{pp}$.

10 Recherches effectuées par nous-même ou sous notre direction par les étudiants du Séminaire de maîtrise et doctorat, "Institutions et vie politique du Québec", depuis quelques années. Nous avons pu ainsi dépouiller systématiquement les biographies des hommes politiques du Québec à l'Assemblée législative et au Conseil législatif pour la période 1867-1966, de même que celles des députés du Québec à la Chambre des communes pour la période 1891-1962. Certaines recherches furent conduites aussi sur le personnel politique de municipalités comme Québec, Trois-Rivières, Sherbrooke pour la période $1900-1965$ et auprès de maires d'une centaine 
Nous nous sommes déjà expliqué ailleurs sur l'orientation que nous entendions donner à cette étude du personnel politique québécois ${ }^{11}$. Dans les quelques pages qui vont suivre, ce sont certaines hypothèses que nous voudrions soumettre concernant la question: quels furent les principaux caractères du personnel politique canadien-français de 1867 à aujourd'hui? principalement quel milieu social fut le sien, et quels changements l'affectèrent au cours de cette longue période? Nous disons hypothèses et non pas conclusions fermes, d'une part parce que l'état actuel de nos recherches sur le personnel politique ne nous permet pas encore d'être trop affirmatif, et pourrons-nous jamais l'être; d'autre part parce que l'état des recherches sur les autres catégories dirigeantes du Québec de même que sur les institutions, les partis, les idéologies et surtout les classes sociales nous invitent à la plus grande prudence. S'il peut être enivrant de s'avancer en terre inconnue ou à peine explorée, on ne peut s'empêcher d'être quelque peu craintif. Ajoutons enfin que les réponses que nous pouvons apporter à la question: "qui furent nos hommes politiques?" n'ont un véritable intérêt que dans la mesure où elles nous permettront de mieux poser une seconde question: "quels rôles a joués cette élite compte tenu du contexte politique, économique, social et culturel dans lequel elle avait à agir?"

Après avoir décrit ce que nous pouvons appeler l'expansion du personnel politique canadien-français du Québec depuis 1867, nous tenterons de le caractériser du point de vue social, d'indiquer quels furent les changements en tentant de marquer les différences existantes entre le niveau provincial et fédéral mais peut-être encore davantage entre les deux groupes fonctionnels qui constituent ce personnel, les ministres et les simples députés. Nous proposerons en terminant une hypothèse sur le rôle qu'a pu jouer ce personnel de 1867 à 1967 à la lumière des caractères que nous aurons décrits.

de municipalités disséminées sur tout le territoire du Québec. Nous tenons ici à exprimer publiquement à nos étudiants toute notre reconnaissance. 11 Robert Boily, Commentaire d'un exposé de M. Jean-Charles Bonenfant sur "L'évolution du statut de l'Homme politique canadien-français", dans Le Pouvoir dans la société canadienne-française, 125-129. 


\section{L'expansion $d u$ personnel politique canadien-français}

La Confédération eut parmi bien d'autres effets celui de permettre une expansion du personnel politique canadien-français en doublant pour ainsi dire les possibilités par la création d'un système politique québécois doté de deux chambres à côté d'un système fédéral, lui aussi bicaméral, et auquel participaient les Québécois. Il faudrait mentionner aussi comme autres facteurs susceptibles d'avoir facilité cette expansion, les modifications survenues notamment en 1875, 1895 et finalement en 1912 concernant le droit de vote et l'éligibilité ${ }^{12}$ ainsi que les modifications survenues à l'intérieur de la province entre Français et Anglais, changements qui eurent pour effet de rendre la plupart des comtés du Québec de plus en plus massivement français. ${ }^{13}$ Peutêtre faudrait-il tenir compte aussi d'un déplacement d'intérêt politique chez les Québécois de langue anglaise avec la création d'un gouvernement central majoritairement anglo-saxon.

Jusqu'à la Confédération et depuis le premier moment où des conseils et assemblées furent créés après la conquête, les Canadiens français n'eurent jamais une représentation qui correspondait à leur importance numérique. En 1774, sous la constitution de l'Acte de Québec, quelques "Canadiens" purent faire leur entrée au Conseil exécutif et au Conseil législatif créé par cette nouvelle constitution. Ils formaient le tiers du premier et environ $40 \%$ du second, certains individus siégeant d'ailleurs dans les deux conseils ${ }^{14}$. L'Acte constitutionnel de 1791 introduisit dans le système politique de 1774 un nouvel élément, une assemblée législative élue. 'Toutefois, même si les Canadiens français formaient alors $94 \%$ de la population ils n'obtinrent que $68 \%$ des sièges, demeurant en minorité dans les deux conseils qui détenaient la réalité du pouvoir ${ }^{15}$. L'Acte d'Union, dont le

12 Jean et Marcel Hamelin, Les Mœurs électorales dans le Québec de $1791 d$ nos jours (Les Editions du Jour, Montréal, 1962), 124 pp., particulièrement les chapitres IV, V, VI.

13 Nathan Keyfitz, "Population Problems", dans French-Canadian Society, 216-244, plus particulièrement 217-224.

14 Desjardins, Guide parlementaire historique de la province de Québec, de 1792 à 1902 (Québec, s. éd., 1902), $396 \mathrm{pp}$.

15 Pierre Elliott Trudeau, op. cit., 242. 
but avoué était l'intégration des "Canadiens" au monde anglosaxon, les plaça de nouveau en minorité à l'Assemblée législative, constituée d'une part des députés du Haut-Canada, tous anglais, et d'autre part des représentants du Bas-Canada, ceux-ci incluant des députés de langue anglaise, chacune des députations des deux provinces possédant un nombre égal de membres.

Pendant toute cette période, à aucun moment les Canadiens français ne furent en majorité dans l'un quelconque des organes politiques possédant la réalité du pouvoir; et lorsqu'enfin la seule assemblée où ils formaient un bloc assez important obtint la responsabilité ministérielle ils ne purent que partager un pouvoir qui pourtant les concernait de très près, un partage qui devenait de plus en plus intolérable à l'élément anglo-saxon du HautCanada et qui amena Brown et ses amis à parler, sans aucune gêne, de la "French domination" 16.

L'obtention en 1867 d'un système politique autonome pour le Québec va donc permettre aux Canadiens français de profiter des effets du rapport démographique qui leur est de plus en plus favorable à l'intérieur des frontières de la province. De 1867 à 1967 non seulement les Canadiens français seront-ils majoritaires au sein des divers organes politiques québécois mais ils prendront peu à peu toute la place. Au niveau fédéral une même progression s'opérera au sein de la représentation québécoise à la Chambre des communes, bien que nous ne puissions pas attacher à cette progression la même signification. ${ }^{17}$

16 Thomas Chapais, Cours d'histoire du Canada, 1861-1867 (Valiquette, Montréal, 1934), $321 \mathrm{pp}$., voir $84,85,86$.

17 Formant en 1867, lors de la première législature qui suivit l'entrée en vigueur de la Confédération, environ $70 \%$ de l'Assemblée législative, les députés canadiens-français atteindront $80 \%$ au tournant du siècle et $90 \%$ peu avant la seconde guerre mondiale. Depuis, la représentation canadiennefrançaise s'est maintenue à ce taux quoiqu'à certains moments l'élément français atteignit près de $93 \%$ comme en 1944. Compte tenu de l'importance numérique des Québécois de langue anglaise dans plusieurs comtés montréalais ce taux de $93 \%$ représente en quelque sorte un maximum pour une très longue période. Si au cours des cinquante premières années de la Confédération la représentation française au Conseil législatif est comparable à celle de l'Assemblée législative $(70 \%)$ peu de changements semblent s'être manifestés puisque pour la seconde période soit de 1917 à aujourd'hui leur représentation n'atteint que 74\%. (En 1962 il y avait 4 conseillers de langue anglaise sur 24). 
On pourrait croire qu'aussi fortement implantés dans les deux chambres et surtout dans la première, l'Assemblée législative, les représentants de langue française se retrouveraient tout aussi nombreux au sein de l'organe dominant de notre système politique, le Conseil des ministres. Cette impression semble confirmée si on examine la liste des premiers ministres du Québec de 1867 à nos jours: deux premiers ministres seulement furent de langue anglaise et tous deux avant le début du XXe siècle, John Jones Ross en 1834 et Edmund James Flynn en 1896. Encore qu'il faille ajouter que l'un des deux avait une mère canadienne-française, qu'ils firent tous deux leurs études secondaires et supérieures dans des institutions canadiennes-françaises et qu'ils épousèrent des Canadiennes françaises. Cependant, si effectivement les grands tribuns, qui tour à tour furent premier ministre et incarnèrent aux yeux de la population le pouvoir politique du Québec, furent presque tous de langue et d'ethnie françaises, ceux qui par leur poste et la tradition politique formaient avec le premier ministre l'organe de décision, furent jusqu'au tournant du siècle très souvent des Anglo-Saxons. En effet, jusqu'au moment où les libéraux s'implanteront au pouvoir avec Marchand, l'élément anglo-saxon du Québec est fortement représenté au Conseil des ministres; bien au-delà de son importance numérique puisqu'ils forment la plupart du temps entre 40 et $50 \%$ des membres des divers gouvernements. De plus, pendant cette période, les Canadiens anglais détiennent des charges ministérielles importantes comme: commissaire des terres, trésorier, commissaire des cheming de fer mais aussi ministre de l'agriculture et procureur général. A partir du premier gouvernement Marchand et avec l'occupation du pouvoir par les libéraux, les Canadiens anglais du Québec seront de moins en moins importants en nombre au Conseil des ministres; ils représenteront $25 \%$ des divers gouvernements libéraux du début, puis $12 \%$ vers la fin du régime Taschereau et enfin depuis 1944 à peine $10 \%$. Cela veut dire que depuis 1944 on ne retrouve au Conseil des ministres qu'un ou deux représentants de l'élément anglo-saxon, qui détinrent sous Duplessis presque continuellement le ministère des mines et, sous Lesage, celui du revenu, 
comme c'est aussi le fait dans l'actuel gouvernement de $\mathbf{M}$. Johnson.

Si donc on laisse de côté le Conseil législatif, dont le rôle à partir de la fin du XIXe siècle devient secondaire, on constate que les Canadiens français sont devenus peu à peu les seuls détenteurs des sièges à l'Assemblée législative et au Conseil des ministres bien qu'à ce dernier niveau l'évolution ait été plus lente et que certains ministres anglo-saxons détiendront souvent des ministères susceptibles de les faire entrer en contact avec les intérêts industriels anglais ou américains qui exploitent les richesses naturelles de la province. ${ }^{18}$ Dès 1867 et pendant toute cette période le poste le plus prestigieux et politiquement le plus important ${ }^{19}$ sera, presque sans interruption, détenu par un Canadien français incarnant en sa personne - le phénomène de personnalisation du pouvoir est très ancien au Québec - le pouvoir provincial, permettant peut-être ainsi d'entretenir l'image d'un véritable pouvoir canadien-français. Avec le XXème siècle, les Canadiens français s'étaient donc emparés, physiquement tout au moins, de l'appareil politique provincial.

Au niveau fédéral la même expansion du personnel politique canadien-français se retrouve avec peut-être un peu plus de lenteur au début. ${ }^{20}$ Des Canadiens français furent ministres très souvent et même premier ministre à deux reprises (Laurier et Saint-Laurent). Toutefois, il faut dire que si dans la plupart des gouvernements les ministres du Québec furent majoritairement canadiens-français, surtout à partir de la fin du XIXème siècle, ils détinrent en général des ministères secondaires. On leur confiait soit un ministère possédant un prestige propre à flatter

18 Il faut dire aussi que des ministres canadiens-français remplirent ce rôle avec beaucoup de talent et beaucoup de zèle, sans parler de certains premiers ministres.

19 Quant au poste constitutionnellement le plus prestigieux, celui de lieutenant-gouverneur, deux Canadiens anglais l'occupèrent: Charles Fitzpatrick, de 1918 à 1923 et Henry George Carroll, de 1929 à 1934.

20 Les représentants canadiens-français atteignent un taux de $70 \%$ seulement, à la fin du XIXème siècle, soit de 1891 à 1904 mais dès la période suivante soit de 1909 à 1911 ils représentent $75 \%$ de la députation du Québec, $80 \%$ en $1925,85 \%$ en 1930 et près de $90 \%$ en 1962 . 
l'électorat du Québec comme celui de la justice, soit un ministère susceptible de susciter de "l'intérêt" comme le ministère des postes ou celui des travaux publics. ${ }^{21}$

Ainsi au fédéral comme au provincial les hommes politiques canadiens-français en sont arrivés à occuper la très grande majorité des sièges du Québec, mais au fédéral ils demeurent une minorité dont le rôle au niveau du gouvernement n'a pu, jusqu'aux toutes dernières années, qu'être très limité, si on s'en tient comme critère, et il a son importance, à l'aspect numérique. Personnel politique canadien-français et personnel québécois en sont venus pratiquement à s'identifier. Au niveau des symboles et de l'utilisation de ces symboles le phénomène est d'importance.

\section{Elite politique et classes sociales au Québec}

S'il est relativement facile de parler de classe ouvrière et de classe paysanne, quoique dans ce dernier cas l'occupation ne soit pas toujours un indice sûr de l'appartenance à ce qui serait une classe paysanne, il est beaucoup plus difficile de stratifier le reste de la population. Bien sûr, on parle de classes moyennes, de classes dirigeantes ou de haute-bourgeoisie, traduisant ainsi beaucoup plus des différences de statut individuel qu'une véritable stratification en classes sociales. Une telle stratification pose toute une série de problèmes théoriques et méthodologiques qui ont fait l'objet de nombreuses études. ${ }^{22}$ Cette difficulté, Porter l'a rencontrée dans son étude de la société canadienne. ${ }^{23}$ Nous ne pouvons que nous y buter nous-mêmes

21 Quant à l'importance numérique des Canadiens français au sein des divers gouvernements nous n'avons des données que pour la période de 1917-1962: pendant cette période des Canadiens français du Québec ont détenu 26 fois un poste de ministre avec portefeuille sur 338 possibilités et 12 fois un poste de ministre sans portefeuille sur 37 possibilités. Il ne s'agit pas de se demander si cette représentation était juste ou injuste, il s'agit plutôt de constater qu'elle faisait d'eux une minorité détenant des postes peu importants.

22 Un excellent exposé de tous ces problèmes se trouve dans Leonard Reissman, Les classes sociales aux Etats-Unis (P.U.F. 1963), 398 pp.

23 John Porter, The Vertical Mosaic (University of Toronto Press, 1965), 626 pp., voir 3 à 28. 
si nous voulons rattacher les hommes politiques à leur milieu social, à leurs classes sociales d'appartenance; rattachement qui s'impose pourtant si nous désirons réellement apporter à la question "Qui gouverne au Québec"? une réponse valable.

Une première approche peut nous permettre de montrer à quel point les hommes politiques du Québec ont été et demeurent peu représentatifs de la population qu'ils gouvernaient, de mettre en relief à quel point ils proviennent et continuent de provenir d'un étroit secteur de la population, constituant ainsi véritablement une minorité, une élite: celle qui consiste à étudier le niveau d'instruction et l'occupation de ce personnel. Cette dernière donnée pouvant d'ailleurs servir de critère objectif de rattachement social par le revenu qu'elle traduit et de critère subjectif par le prestige qui y est attaché. Une deuxième approche, en prenant appui sur ces deux données mais en y ajoutant d'autres facteurs de rattachement comme l'origine familiale, la naissance, la puissance économique, tendrait à stratifier ce personnel en cinq catégories ou classes en dissociant d'une part ceux qui appartiendraient à ce que nous pouvons appeler la haute-bourgeoisie canadienne-française, des classes moyennes, ouvrières et paysannes et d'autre part en distinguant au sein de ce vaste groupe hétérogène des classes moyennes un niveau supérieur et un niveau inférieur que nous appellerons la classe moyenne supérieure et la classe moyenne inférieure. La distinction entre haute-bourgeoisie et classes moyennes s'impose si on veut véritablement déboucher sur une analyse des catégories dirigeantes au Québec de même que s'impose la distinction de deux niveaux à l'intérieur du groupe "classes moyennes", premièrement parce que la très grande majorité des hommes politiques s'y rattache comme nous allons le voir et d'autre part parce qu'il serait dangereux de confondre dans le même groupe les membres des professions libérales jouissant d'un revenu, d'un prestige, d'une instruction qui fait d'eux une catégorie vraiment à part, et les marchands, boutiquiers, commerçants et petits administrateurs qui, s'ils sont indéniablement avec les membres de professions libérales, les notables locaux et l'un des groupes composants des 
classes moyennes, ne peuvent être confondus avec le premier groupe et ne le sont d'ailleurs pas par la population. ${ }^{24}$

\section{Formation classique et pratique juridique}

Les subtils débats qui animaient l'Assemblée législative ou le Conseil législatif sous l'Union au moment de la discussion du régime seigneurial, le caractère électif du Conseil législatif ou encore les débats byzantins entre radicaux et ultramontains après 1867, débats au cours desquels l'ensemble des idéologies politiques était passé en revue, contrastaient singulièrement avec l'état général de notre société, cette société si démunie sur le plan de l'instruction. Société longtemps non-instruite mais qui attacha toujours à l'instruction une très grande valeur, à la fois parce qu'on lui présentait la culture et l'instruction comme son domaine propre d'expansion et d'expression, ${ }^{25}$ mais peutêtre aussi, peut-être davantage, parce que consciemment ou inconsciemment elle pressentait que c'était là la clé de la promotion individuelle et collective.

Si aujourd'hui la tenue des débats en Chambre continue de contraster avec l'état de la population - bien que parfois on soit tenté de croire que les rapports soient maintenant inversés ! - il reste que tout au long de ces cent années, à l'instar il est vrai de beaucoup d'autres sociétés, le Québec fut gouverné par des gens instruits, possédant majoritairement une formation universitaire et massivement une formation secondaire. ${ }^{26}$ C'est dire que la très grande majorité de nos hommes politiques a reçu une formation que seule une minorité pouvait recevoir au Québec; c'est dire aussi que la majorité de nos hommes politiques a reçu une formation homogène au sein des séminaires

24 Il est à noter que le rattachement des hommes politiques à l'une ou l'autre classe s'est fait à partir d'une analyse de la situation qui était la leur au moment de leur première élection ou nomination.

25 Cela faisait partie du messianisme de Mgr Paquet.

26 Dès 1867 , plus de $60 \%$ des députés possédaient au moins une formation secondaire et à partir de 1886 , au-delà de $40 \%$ des membres de chacune des législatures sont de formation universitaire, ce taux variant par la suite entre $40 \%$ et $50 \%$ (ce taux a toujours eu tendance à être plus élevé depuis 1917 lorsque les libéraux sont au pouvoir) pour atteindre, aux dernières élections, celles du 5 juin 1966, un taux de $66 \%$. 
et collèges de la province, séminaires et collèges qui étaient tous des institutions privées et par là réservées à une élite, distribuant une formation de type humaniste sous le contrôle étroit du clergé et remplissant une triple fonction d'intégration, de déracinement et de mobilité sociale. ${ }^{27}$ De 1867 à aujourd'hui, peu d'hommes politiques eurent uniquement une formation dans des écoles publiques de niveau primaire ou secondaire; ils dépassèrent rarement le tiers de la représentation. Ce qu'il faut noter, c'est le caractère constant de ces tendances au cours de ce siècle de vie politique. La diminution récente du nombre de députés de formation primaire répond dans une certaine mesure à des changements survenus dans la population - en 1966 ils ne sont plus que quelques-uns - mais cette diminution a profité tout autant sinon plus à la catégorie des députés de formation supérieure ou universitaire qu'à ceux de formation secondaire. ${ }^{28}$

Le contraste entre gouvernants et gouvernés est encore plus saisissant si on s'arrête aux seuls membres du Conseil des ministres pendant cette période. De 1886 à 1900, des 16 ministres canadiens-français sur lesquels nous avons obtenu des renseignements et qui représentent d'ailleurs la presque totalité des ministres canadiens-français de cette époque, 15 avaient reçu une formation universitaire. De 1917 à 1962, sur les $103 \mathrm{mi}-$ nistres que nous avons pu étudier, ministres avec portefeuille (74) ou ministres d'État (29), 63 avaient reçu une formation universitaire et 12 autres une formation secondaire dans des séminaires ou collèges. Notons enfin qu'un seul des premiers ministres depuis 1867 n'a pas reçu une telle formation.

Qu'il s'agisse du Conseil législatif ou de la Chambre des communes on retrouve la même importance des hommes poli-

27 Maurice Tremblay, "Orientations de la pensée sociale”, dans Jean-C. Falardeau, éd. Essais sur le Québec contemporain (Québec, P.U.L. 1953), 193-208. 1966 .

28 Niveaux d'instruction des membres de l'Assemblée législative 1867-

\begin{tabular}{|c|c|c|c|c|c|}
\hline & 1867 & $1892-1900$ & $1908-1916$ & $1919-1962$ & 1966 \\
\hline $\begin{array}{lllll}\text { Primaire } & \ldots & \ldots & \ldots \\
\text { Secondaire } & . . & \ldots & . \\
\text { Supérieur }\end{array}$ & $\begin{array}{l}37 \\
38 \\
25\end{array}$ & $\begin{array}{l}21 \\
23 \\
56\end{array}$ & $\begin{array}{l}12 \\
35 \\
53\end{array}$ & $\begin{array}{l}19 \\
33 \\
48\end{array}$ & $\begin{array}{r}1 \\
33 \\
66\end{array}$ \\
\hline
\end{tabular}


tiques de formation universitaire et de ceux ayant reçu une formation dans des institutions privées. $\mathrm{Au}$ Conseil législatif, c'est près de $65 \%$ des conseillers sur lesquels nous avons eu ce renseignement (près de 70\% d'entre eux) qui reçurent ainsi une formation universitaire pour la période $1867-1917$ et $70 \%$ pour la période 1917-1965 (ce renseignement nous était connu pour $80 \%$ d'entre eux). A la Chambre des communes les députés canadiens-français furent là aussi très majoritairement des hommes d'un niveau d'instruction supérieur à celui de la population, et d'une certaine manière ce décalage était encore plus accentué qu'au provincial ${ }^{29}$ par suite du pourcentage beaucoup plus élevé d'universitaires et, beaucoup plus faible, de députés de formation primaire, et cela dès le XIXème siècle.

Quels que soient donc les divers niveaux de gouvernement ou les divers types d'organes, le même type d'homme politique a dominé, celui qu'avaient formé le collège classique et l'université. Si quelques représentants ayant reçu une formation primaire ou encore secondaire dans des écoles publiques ont eu une certaine importance, depuis le début du XXème siècle surtout à l'Assemblée législative, ils furent avant tout de simples "backbenchers".

Un nombre aussi élevé d'hommes politiques canadiens-français passés par les universités ne peut que traduire la place dominante au sein de notre personnel politique des membres de professions libérales. En effet, dès la première législature, celle de 1867 , près de $70 \%$ des membres de l'Assemblée législative appartiennent au groupe des professions libérales à côté de quelques hommes d'affaires, industriels ou marchands (12\%), et des individus déclarant comme profession, cultivateurs. Par la suite, à mesure que les représentants du commerce et de l'industrie prendront de l'importance - les hommes d'affaires deviendront à partir de la législature de 1908 plus nombreux

${ }^{29}$ Niveaux d'instruction des députés du Québec à la Chambre des communes 1891-1962.

\begin{tabular}{|c|c|c|c|}
\hline & $1891-1904$ & $1904-1917$ & 1917-1962 \\
\hline 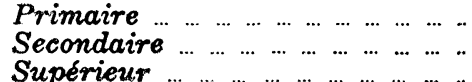 & $\begin{array}{l}11 \\
35 \\
54\end{array}$ & $\begin{array}{r}5 \\
17 \\
78\end{array}$ & $\begin{array}{r}3 \\
20 \\
77\end{array}$ \\
\hline
\end{tabular}


que les cultivateurs et propriétaires terriens et constitueront pratiquement toujours près de $40 \%$ des diverses législatures les membres des professions libérales tout en demeurant majoritaires verront leurs effectifs quelque peu réduits, à environ la moitié du personnel. ${ }^{30}$

Avocats, médecins, notaires ont au cours des cinquante premières années été à peu près les seuls représentants des professions libérales parmi les députés. À partir du début du siècle, d'autres professions seront représentées telles l'agronomie, la médecine vétérinaire, la comptabilité, etc., mais sans jamais atteindre l'importance des professions traditionnelles du Québec qui constitueront toujours au moins $70 \%$ de ce groupe. Les avocats tout particulièrement, comme dans bien d'autres pays, ont toujours formé le bloc le plus imposant, soit généralement un peu plus de la moitié de cette catégorie et environ le quart de tout le personnel politique de l'Assemblée législative. Seuls les médecins ont eu une certaine importance soit, en moyenne, pendant toute cette période, $20 \%$ du groupe des professions libérales. Avocats et médecins sont depuis bien avant 1867 les figures les plus connues de la politique provinciale. Ils ont toujours représenté deux professions dont le prestige était immense dans la population; les premiers, avant tout, un prestige social, les seconds un prestige moral qu'ils partageaient avec les membres du clergé et qui faisait d'eux des candidats toujours redoutables. Quant aux notaires, souvent plus importants que les médecins avant 1900, ils ne seront plus représentés par la suite que par quelques individus.

Longtemps les avocats, médecins, notaires constitueront à eux seuls les notables locaux, les "leaders" naturels de la société québécoise. C'est en quelque sorte un statut qu'ils ont acquis au cours du début du XIXème siècle. Même lorsque par suite de l'industrialisation, de l'expansion du petit commerce et même

30 Si en 1867 ils sont $70 \%$ de la législature, dès 1886 ils ne sont plus que $50 \%$ et à partir du début du XXème siècle leur représentation oscillera entre $40 \%$ et $50 \%$, plus souvent d'ailleurs $50 \%$, notamment quand les libéraux sont au pouvoir. Ils sont $61 \%$ de la législature issue des élections du 5 juin 1966. 
d'une certaine diversification de la population active, les commerçants, marchands et petits industriels rivaliseront avec eux sur le plan local, notamment au sein des conseils municipaux, il sera plus difficile à ces derniers, il leur faudra plus de temps pour accéder au statut d'homme politique. Ils ne viendront à la politique qu'une fois seulement que leur réussite en affaires aura fait d'eux des vedettes locales. Même maintenant il demeure plus facile à un jeune avocat par exemple d'entrer en politique et d'y réussir, comme s'il bénéficiait encore dans la psychologie collective du prestige attaché autrefois aux membres des professions libérales alors qu'ils constituaient à peu près la seule élite reconnue par la société québécoise. Socialement, le système de valeurs a pu changer; politiquement, il semblerait que les valeurs anciennes demeurent encore effectives, comme si dans ceite société nouvelle qu'est devenu le Québec l'électeur n'avait pas modifié ses critères d'appréciation, à moins que ce soit l'homme politique traditionnel qui ait su préserver son statut.

Si le personnel politique du Québec à l'Assemblée législative fut à $90 \%$, et le demeure, formé de représentants de professions libérales, la plupart du temps traditionnelles, et de représentants du secteur des affaires, surtout à partir de 1900, ces deux groupes n'ont pas eu la même possibilité d'accès aux fonctions dirigeantes, c'est-à-dire aux fonctions ministérielles, et cela semblerait confirmer l'hypothèse précédente. Que les ministres avant 1900 soient surtout des membres de professions libérales ne peut pas tellement surprendre, vu leur plus grande importance à l'Assemblée législative, surtout dans les toutes premières législatures. ${ }^{31}$ Cependant de 1917 à 1962, malgré l'augmentation des représentants du secteur du commerce et de la petite industrie dans la compétition politique, les membres de professions libérales et surtout les avocats continueront de dominer le Conseil des ministres. ${ }^{32}$ Encore faut-il ajouter que sauf trois exceptions

${ }^{31}$ De 1886 à 1900 , sur 14 ministres canadiens-français étudiés de ce point de vue, il y avait 10 avocats, 2 notaires et seulement 2 représentants du monde des affaires.

32 Sur 118 ministres étudiés, très majoritairement canadiens-français, 72 provenaient des professions libérales et 29 seulement du milieu des 
(Boucher de Boucherville, Godbout, Barrette) le premier ministre fut toujours un avocat.

Ces caractères de la représentation à l'Assemblée législative sont aussi ceux du Conseil législatif et de la députation canadienne-française à la Chambre des communes, à quelques nuances près. En effet, si dans ces deux assemblées, membres des professions libérales et hommes d'affaires dominent largement, au Conseil législatif, ${ }^{33}$ ils se répartissent un peu plus également et les représentants du secteur des affaires proviennent surtout de la moyenne et de la grande industrie, tandis qu'à la Chambre des communes les membres de professions libérales sont encore plus importants qu'à l'Assemblée législative. ${ }^{34}$

\section{L'élite politique canadienne-française: haute-bourgeoisie et classe moyenne supérieure}

Les caractères que nous venons de décrire traduisent au fond une réalité fréquente et souvent analysée dans plusieurs sociétés politiques occidentales ${ }^{35}$; donc rien qui ne doive trop nous surprendre. Toutefois, ce qu'il faut quand même souligner, c'est dans une grande mesure la constance, la stabilité de ce personnel politique dans ses caractères constituants: il fut et demeure avant tout un personnel de notables provenant essentiellement du secteur des professions libérales et des affaires et peu sensible à l'émergence de nouvelles élites ou à la trans-

affaires, les autres étant ouvriers ou paysans. Les avocats à eux seuls ont représenté près de $73 \%$ de ces ministres membres de professions libérales et $45 \%$ de l'ensemble des ministres, c'est-à-dire un pourcentage bien supérieur à leur importance numérique au sein du personnel politique; de plus ils furent surtout des ministres avec portefeuille.

33 Les membres de professions libérales ont représenté $48 \%$ des conseillers législatifs de 1867 à 1915 et $41 \%$, de 1915 à 1965; ceux du secteur des affaires $35 \%$ de 1867 à 1915, 39\%, de 1915 à 1965 .

$3452 \%$ de $1891-1904,60.7 \%$ de $1909-1911$ et $65 \%$ de $1917-1962$. Les avocats représentaient, de 1891-1909, $70 \%$ d'entre eux, 60\%, de 1904-1911 et $68 \%$, de 1917-62. Au cours de cette dernière période leur représentation a varié entre 25 à $56 \%$ de l'ensemble de la députation québécoise. Même si ces résultats concernent l'ensemble de la députation, ils sont représentatifs de la situation des Canadiens français, ceux-ci étant surtout des membres de professions libérales.

35 Voir à ce sujet les études publiées dans La Revus Internationale des Sciences Sociales, "La Profession Parlementaire" (Vol. XIII, n 9, 1961). 
formation des classes moyennes notamment depuis 1945. S'arrêter là serait cependant négliger un fait très important: s'il y a stabilité dans le niveau d'instruction et dans le type d'occupation, il $\mathrm{y}$ a eu changement dans l'appartenance sociale des hommes politiques du Québec et par suite dans le type d'homme politique et peut-être même dans la fonction que remplissait la carrière politique.

Au moment où s'amorce ce centenaire de vie politique, la société canadienne-française a déjà commencé à être modifiée et le sera encore davantage dans les années qui suivront, sous l'effet de l'industrialisation. Cependant, au point de vue de la structure sociale, le caractère dominant demeure et demeurera d'ailleurs pour la majeure partie de cette période ${ }^{36}$ l'importance de la masse, l'étroitesse des classes moyennes et la faiblesse numérique de ce qui constitue la haute-bourgeoisie canadiennefrançaise ou de ce qui en tient lieu. Une masse constituée presque essentiellement en 1867 de paysans ou du moins de ruraux et auxquels s'ajoutèrent à mesure que la révolution industrielle s'élargissait au Québec, les ouvriers et les salariés en général, de plus en plus nombreux, au point de devenir rapidement l'élément majoritaire. Tout au cours de cette période, les classes moyennes varieront peu quant à leur importance relative au sein de la collectivité canadienne-française et quant à leur composition: elles demeureront presque essentiellement constituées de représentants des grandes professions libérales et de la petite-bourgeoisie d'affaires, marchands et petits industriels de province. Quant à la haute-bourgeoisie, elle est formée des quelques industriels et hommes d'affaires ou financiers participant au pouvoir économique, des représentants de la classe seigneuriale, liés la plupart du temps à des familles anglaises, et des représentants de quelques familles bourgeoises anciennes possédant un certain prestige en politique ou dans la magistrature, comme les Taschereau, les Tessier, les Chapais. Ces derniers représentants de ce qui serait la haute-bourgeoisie canadienne-française viennent en général, comme les Papineau et plusieurs autres, de la nouvelle bourgeoi-

36 Jacques Brazeau, "Quebec Emerging Middle Class", in French Canadian Socisty, 319-327. 
sie qui se forma à la fin du XVIIIème et au début du XIXème siècle à partir des professions libérales comme le droit et la médecine, et connut son expansion dans la politique, la magistrature et les affaires.

$\mathrm{Du}$ point de vue des relations classes sociales-pouvoir politique, trois faits caractériseraient principalement le personnel politique canadien-français: premièrement, c'est l'absence ou la faible représentation de deux classes qui de tout temps ont constitué la masse de la population, la classe paysanne et la classe ouvrière; deuxièmement, le contrôle des institutions politiques québécoises et du personnel politique canadien-français jusqu'aux années 1920 environ par une classe, la haute-bourgeoisie; troisièmement, son remplacement non seulement par les représentants de la classe moyenne supérieure mais par un type d'hommes politiques différents, disons l'homme politique de métier. Le régime Taschereau constituerait le régime de transition au point de vue de la substitution des élites politiques. C'est à la fin de ce régime et avec les deux gouvernements Godbout que les représentants des classes moyennes arrivent au pouvoir; c'est avec Duplessis qu'elles s'y installent et l'exploitent. Sous Lesage et Johnson, ce sont ces mêmes classes moyennes qui dominent l'Assemblée législative mais d'une manière plus forte encore. Depuis 1962 cependant, mais surtout depuis les élections de 1966, on peut discerner l'apparition d'hommes liés à ce que l'on commence à appeler les nouvelles classes moyennes canadiennes-françaises, provenant de secteurs dont l'expansion est récente au Québec mais qui traduisent le caractère de plus en plus industriel et technocratique de notre société. Les Masse, les Tremblay, les Lévesque, les Lefebvre, les Bourassa en sont les exemples les plus connus.

\section{La sous-représentation de la masse}

Peuple de paysans et d'ouvriers modestes, le Canada français confia très rarement la défense de ses intérêts à des représentants de ces deux classes qui pourtant ont toujours formé la majorité du peuple canadien-français et fait de lui un prolé- 
tariat ethnique à l'intérieur du Canada ${ }^{37}$. Si l'absence de représentants de la classe ouvrière avant 1900 n'étonne pas tellement, le Québec entrant pour ainsi dire "à reculons" dans la civilisation industrielle et urbaine, la faiblesse de la représentation paysanne pendant la même période, soit à un moment où le Québec est encore fortement rural, étonne davantage, encore plus peut-être que l'absence à peu près totale d'ouvriers ou de salariés au cours de la seconde moitié de ce centenaire de vie politique, quand on songe à quel point le mode de vie rural a été tenu pour privilégié jusqu'aux années trente alors que l'on ne manifesta que beaucoup de crainte et surtout de mépris pour le mode de vie urbain et la condition ouvrière..$^{38}$

Cette faiblesse de la représentation de la classe ouvrière et de la classe paysanne au sein des assemblées parlementaires

37 Everett C. Hugues, "French and English Canadians in the Industrial Hierarchy of Quebec", in Canadian Society (ed. B. Blishen, F. Jones, F. Nargele, J. Porter, Macmillan, 1964), 423-435. - John Porter, op. cit., 73-91.

38 De 1867 à 1900 environ, le taux moyen de la représentation de la classe paysanne, si on prend pour critère l'occupation de cultivateurs, est d'environ $13 \%$ au sein de l'Assemblée législative; encore faut-il préciser qu'il s'agit là d'un maximum et que très souvent à cette époque il s'agissait de propriétaires terriens. Après 1886 , si leur représentation s'éleva quelque peu, elle ne dépassa pas $20 \%$ pour se maintenir après 1900 à un taux moyen de $10 \%$. La forte implantation de l'U.N., en milieu rural, et j'entends par là l'origine rurale de ses députés, leur permit de conserver jusqu'à 1960 environ une représentation presque aussi importante, relativement, que sous Taschereau, ceci à un moment où le Québec était devenu majoritairement urbain et industrialisé. Depuis 1960, seuls quelques individus représentent cette classe de notre société à l'Assemblée législative. Quant à la classe ouvrière, elle fut à peu près absente de la Chambre basse, seuls quelques représentants réussissant à être élus, notamment entre 1900 et 1916, moment d'une certaine effervescence ouvrière, puis de nouveau entre 1936 et 1948. Des élus de cette dernière période, deux firent une très belle carrière, Antonio Barrette et Maurice Bellemare. Dans les deux cas, il ne s'agissait d'aucune manière d'une représentation de classe.

Au Conseil législatif et à la Chambre des communes, c'est la même faiblesse de représentation. Seuls quelques cultivateurs furent nommés au Conseil législatif ( $8 \%$ des conseillers de 1867-1915 et $11 \%$ de 1915-1965) et, semble-t-il, aucun ouvrier ou collet blanc. A la Chambre des communes on ne trouve de 1891 à 1904 aucun ouvrier et seulement 12 cultivateurs sur 133 députés, 1 ouvrier et 6 cultivateurs sur 112 députés de 1904-1911 et 4 ouvriers, 26 cultivateurs et; un collet blanc sur 896 députés étudiés pour la période 1917-1962. Comme parmi ces députés il y avait des Canadiens anglais, cela fait donc un tout petit nombre de représentants pour les deux classes majoritaires du Québec. 
s'accompagne d'une faiblesse de leur représentation au sein du Conseil des ministres. Pour ne prendre que quelques points de repère et ne retenir que le niveau provincial, disons que de 1886 à 1900 aucun ministre canadien-français n'était un cultivateur intégré à la classe paysanne et, pour toute la période 1917-1962, sur 44 cultivateurs élus (sur un total de 456 personnes analysées), 8 seulement devinrent ministres dont 6 ministres d'État. Il y eut bien 3 ministres ayant déclaré comme occupation, "ouvrier", mais, pour au moins deux d'entre eux. il s'agissait, comme nous l'avons dit, d'hommes politiques de métier.

On peut faire appel à toute une série de facteurs pour expliquer cette sous-représentation du monde paysan et ouvrier et cet éloignement du pouvoir. Contentons-nous ici d'en rappeler quelques-uns. Il faut noter tout d'abord qu'il s'agit là d'un phénomène pratiquement universel, la plupart des études faites. sur des personnels politiques dans les sociétés comparables à la nôtre l'ayant démontré et, entre autres, celle faite par Norman Ward sur le personnel canadien. ${ }^{39}$ Comme dans plusieurs autres sociétés, le monde rural québécois a remis la défense de ses intérêts à ses notables locaux, avocat, médecin, notaire, marchand ou industriel. Il était poussé à cet abandon de la défense de ses. intérêts d'une part par sa condition économique et sociale qui le plaçait en situation de dépendance, d'autre part par le système de valeurs dominant ${ }^{40}$ qui entretenait le respect de l'ordre établi et notamment celui de la structure sociale ainsi que l'acceptation de sa condition. Dominés socialement par les "instruits", les. ruraux le furent politiquement. Quant aux ouvriers, soumis au même système de valeurs, totalement aliénés économiquement, ils avaient en plus l'impression d'être plus ou moins en dehors de la "vraie" société canadienne-française et leur état de pauvreté et d'insécurité ne leur permettait pas facilement d'assumer le

39 Norman Ward, The Canadian House of Common: Representation (University of Toronto Press, 1963), $307 \mathrm{pp}$.

40 Système de valeurs développé et entretenu par cette élite qui les. dominait et les représentait. 
risque d'une élection et d'une carrière politique peu rémunératrice pendant longtemps. ${ }^{41}$

Seul un vaste mouvement de classes à la suite d'une prise de conscience aurait pu permettre à l'une ou l'autre classe d'avoir accès aux postes dirigeants de la politique par l'intermédiaire de quelques-uns de ses représentants. Un tel mouvement, une telle prise de conscience n'a jamais réellement eu de tels effets quand elle eut lieu comme dans les années 1900-1920. C'est que toute tentative d'une prise de conscience de l'une ou l'autre de ces deux classes a toujours été rapidement détournée, contrôlée et plus ou moins tuée par les élites dirigeantes, politiques ou religieuses. ${ }^{42}$ D'autre part, les groupes représentant les intérêts de ces deux secteurs économiques et sociaux ont toujours manifesté beaucoup de méfiance à l'égard de la politique et particulièrement des partis politiques. ${ }^{43}$ Restait la formation de partis politiques qui, échappant au conformisme idéologique des deux partis traditionnels, aurait pu puiser en ces milieux l'occasion d'un renouvellement du personnel politique. L'emprise de l'idéologie unitaire, une culture politique déjà ancienne qui entre autres choses a implanté une conception dualiste de la vie politique faisant appel à des fidélités partisanes plutôt qu'à des idéologies, de même que l'action de certains facteurs techniques comme le système électoral et la carte électorale sans parler du caractère censitaire du suffrage pendant une partie importante de la période étudiée ${ }^{44}$ ont toujours, jusqu'à maintenant, rendu extrêmement difficile la naissance et la survie de tiers-partis susceptibles, par suite des intérêts qu'ils défendaient, de puiser en ces milieux. ${ }^{45}$

41 Encore en 1946 le montant total que pouvait recevoir un député pour une session d'au moins 30 jours était de $\$ 4,000$.

42 Gérald Fortin, "Milieu rural et milieu ouvrier, deux classes virtuelles", Recherches sociographiques, Les classes sociales au Canada français, VI: I (1965).

43 Seule la F.T.Q. a des liens assez étroits et publics avec un parti politique, le N.P.D.

44 En fait, jusqu'au début du XXème siècle.

45 Robert Boily, "Aspects politiques des systèmes électoraux", PartiPris, vol. 3 no 7 (février 1966) : 8-29. 


\section{L'accès des classes moyennes au pouvoir}

On le voit, leur niveau d'instruction et leurs occupations nous l'avaient déjà laissé entrevoir, l'origine sociale de nos hommes politiques ne doit pas être cherchée dans les secteurs de la population qui incarnaient le mieux le peuple canadien-français mais bien dans les étroites couches supérieures de cette société, constituées principalement de l'élite des professions libérales et des affaires, au sein de ce que nous avons appelé la haute-bourgeoisie et les classes moyennes. C'est au moins $85 \%$ de nos hommes politiques qui doivent être rattachés à ces deux milieux depuis 1867 et, pour les dernières législatures, tant à Ottawa qu'à Québec, au delà de $90 \% .{ }^{46}$ Mais encore une fois, l'instruction et l'occupation nous permettaient déjà de saisir cette appartenance à une élite du personnel politique canadienfrançais. Seule une analyse minutieuse des données, ici les biographies, peut nous permettre de saisir l'importance relative des représentants de la haute-bourgeoisie et des classes moyennes et, à l'intérieur de celles-ci, de la classe moyenne-supérieure et de la classe moyenne-inférieure. Pour cette raison, nous allons maintenant nous limiter surtout au niveau provincial et notamment au Conseil des ministres.

Ce que cette analyse révèle, c'est que de 1867 à 1920 environ une élite politique, qui constitua ce que nous pourrions appeler une classe politique, domina le conseil des ministres et la vie politique du Québec, une élite qui s'alimentait surtout à la haute-bourgeoisie canadienne-française, mais qui par la suite sera remplacée par des individus se rattachant surtout aux classes moyennes et tout particulièrement à la classe moyennesupérieure, formée surtout de membres des professions libéra-

${ }^{46} \mathrm{Ce}$ taux de $85 \%$ est assez représentatif de l'origine sociale des conseillers législatifs pendant toute cette période. A l'Assemblée législative, l'importance plus grande des représentants de la classe paysanne de 1886 à 1900 fait que si ces deux groupes sociaux représentent près de $85 \%$ en $1867,90 \%$ pour la période de $1875-1881$, ils ne représentent plus que 80\% de 1886-1911. D'une manière générale le taux moyen fut de $90 \%$ sous Taschereau et de moins de $85 \%$ sous Duplessis. Depuis 1956 , ces deux groupes représentent plus de $90 \%$, près de $95 \%$ depuis les dernières élections. A la Chambre des communes, ils formaient $88 \%$ du personnel de 1891-1917 et $91 \%$ de 1917-1962. 
les. ${ }^{47}$ Depuis 1867, le Conseil des ministres a été pratiquement sous le contrôle d'avocats originaires de la haute-bourgeoisie ou de la classe moyenne-supérieure. Avant 1917, les représentants de ces deux classes constituaient la presque totalité des ministres canadiens-français et, après 1917 , ils seront $72 \%$ des ministres et sauf une fois, les premiers ministres. L'élite politique canadienne-française, ce fut eux avant tout.

47 Bien que les représentants de la classe moyenne-supérieure constitueront en général les deux tiers des hommes politiques que nous pouvons rattacher aux classes moyennes, il ne faut pas négliger le fait que les représentants de la classe moyenne-inférieure ont connu une expansion considérable au sein du personnel politique québécois depuis 1867. Pratiquement absents des premières législatures et cela jusqu'en 1881 environ ( $8 \%$ du personnel), ils seront $20 \%$ des députés à l'Assemblée législative au début du siècle et en moyenne $30 \%$ tout au long de la période 1917-1967. C'est sous Duplessis que les représentants de cette classe connaîtront leur plus grande expansion $33 \%$ des députés à la fin du régime Duplessis. C'est la participation de plus en plus grande des petits commerçants, marchands, petits administrateurs de toute sorte à la compétition politique, qui explique cette expansion. Ce sont eux qui semblent avoir succédé aux paysans et avoir pris la place des représentants de la classe ouvrière et se retrouver ainsi aux côtés de l'homme politique canadien-français traditionnel, l'avocat.

Comment expliquer l'entrée en politique à partir d'un certain moment des représentants de la classe moyenne-inférieure? L'explication pour nous serait la suivante: sous le régirne Taschereau, la sélection des candidats se serait faite de moins en moins en fonction d'une fidélité à un parti, d'une qualité de militant ou encore par cooptation d'un jeune, membre de la même étude juridique, parent, etc., par le député en place, mais plutôt à partir de l'idée qu'il fallait choisir l'homme ayant le plus de chance d'être élu, c'est-à-dire celui qui était le plus connu, le plus populaire, celui qui, grâce à ses relations, pouvait le plus facilement obtenir le plus grand nombre de voix et devenir l'intermédiaire efficace du parti dans le comté. Ses idées importaient peu. Une fois qu'il était désigné, on mettait tout en cuvre pour le décider. Or, le commerçant, le marchand, le petit industriel local remplissait souvent ces conditions. Il trouvait dans cette carrière nouvelle le prestige que ne lui accordait pas toujours son occupation; on trouvait en lui un individu intégré depuis longtemps à son milieu et généralement très actif au sein des institutions locales, municipales ou sociales.

Ajoutons enfin que si les représentants de cette classe furent surreprésentés à l'Assemblée législative - ils sont loin de constituer $30 \%$ de la population active - ils n'eurent qu'une faible participation au Cabinet d'une manière générale. Ils furent avant tout des soldats plutôt que des capitaines. Avant 1917, ils seront pour ainsi dire absents du cabinet. Après 1917 et jusqu'en 1962, 14\% seulement des représentants de cette classe y accéderont, souvent comme ministres d'Etat. C'est surtout sous le régime Duplessis qu'ils eurent l'accès le plus large: sur 34 ministres canadiensfrançais qui siégèrent au Conseil des ministres pendant cette période, et sur lesquels nous avons des renseignements, 12 appartenaient à cette classe, alors qu'ils furent tout au plus 2 sous Taschereau et 4 sous Godbout. 


\section{Aristocratie et partitocratie}

Cette élite politique, elle fut d'abord et avant tout, et cela jusqu'aux années vingt, constituée des représentants de la hautebourgeoisie canadienne-française ou de ce qui en tenait lieu, avant de devenir essentiellement constituée de représentants des classes moyennes. Mais qui sont ces gens que l'on situe ainsi au-dessus de la classe moyenne-supérieure? Nous serions tentés de répondre: pour la plupart des individus appartenant par la naissance ou le mariage à des familles dominantes de la vie politique québécoise, parfois depuis la fin du XVIIIème siècle, qui ont aussi fourni plusieurs figures marquantes de la magistrature, des familles nouant entre elles un réseau très complexe de liens par le mariage ou encore les études juridiques. C'est de ces familles que Robert Rumilly disait très justement: "Une famille de bonne bourgeoisie, compte un ministre, un haut fonctionnaire fédéral, un juge, un évêque ou au moins un Supérieur de communauté ou un Protonotaire apostolique." 48 Faisaient aussi partie de cette classe politique des individus qui, quoique d'origine modeste, paysanne généralement, avaient accédé par leur instruction et leur occupation à la classe moyenne-supérieure et qui, par leur carrière tant professionnelle que politique, l'adoption des mêmes valeurs et du même genre de vie, avaient réussi à s'introduire au sein de cette élite, comme ce fut le cas pour Mercier. Ajoutons enfin que lorsque ces individus qui avaient connu une ascension aussi rapide, en grande partie grâce à la politique, avaient une descendance, ils se trouvaient alliés très rapidement à des familles plus anciennes.

Ce sont les représentants de cette classe politique qui, au cours des cinquante premières années de la Confédération, dominèrent la scène politique québécoise; ce sont eux qui imposèrent leur système de valeurs, leurs choix politiques. Si une carrière comme celle de Mercier peut faire croire à l'existence d'une grande mobilité sociale, il faut bien voir qu'il s'agit d'une mobilité de type aristocratique. La classe dirigeante se renouvelle en intégrant les nouveaux venus.

${ }^{48}$ Robert Rumilly, op. cit., tome XXV, “Alexandre Taschereau”, 19. 
Ce qu'il faut retenir avant tout, c'est leur importance au sein des Conseils des ministres jusqu'à 1920 environ, ensuite le caractère particulier de leur carrière. N'ayant jamais représenté qu'un infime pourcentage du personnel politique canadien-français de cette période, sauf peut-être dans les toutes premières années, ${ }^{4 \theta}$ ils détiendront près de la moitié des ministères et ils continueront de jouer un rôle important même après 1920 par l'intermédiaire notamment d'une forte personnalité comme celle de Taschereau ou Duplessis. Ce rôle dominant, ils semblent l'avoir rempli dans un style assez particulier. Ils évoluaient très souvent avec une grande aisance et une grande facilité non seulement entre Ottawa et Québec, ${ }^{50}$ ou encore entre les assemblées élues et les assemblées nommées, mais encore entre la politique et la magistrature et vice versa. Ils trouvaient facilement une retraite comme juge, sénateur, conseiller législatif ou encore lieutenant-gouverneur.

Il ne nous est pas possible actuellement de reconstituer tout le réseau de liens qui unissaient les divers membres de cette classe politique entre eux de mêrne qu'il ne nous est pas possible d'analyser les divers gouvernements dans lesquels ils jouèrent un rôle important, ni comment ils étaient en contact avec certaines grandes familles canadiennes-anglaises ou avec certains intérêts financiers dominants, à titre de conseillers juridiques la plupart du temps, ou encore de membres de conseils d'administration. Qu'il nous suffise pour le moment de rappeler quelques noms: Boucher de Boucherville, Taschereau, Mercier, Gouin, Turcotte, Pelletier, Langelier, Gagnon, Dessaulles, Lemieux, Choquette, Tessier, etc. Le lecteur trouvera en renvoi le profil de trois types d'appartenance à cette élite. Ces exemples illustrent

49 Tout au plus $10 \%$.

50 De 1891 à 1904, 25 députés fédéraux du Québec avaient déjà siégé au provincial, dont 17 canadiens-français. De 1904 à 1911, leur nombre tombe à 10 et par la suite leur nombre ne devait jamais être très élevé. Quant aux passages du fédéral au provincial ils furent eux aussi réduits; moins de 15 cas de 1867 à 1967 et 17 environ sur 496 individus étudiés de 1917 à 1962 . Ces passages ont permis très souvent l'accès au cabinet provincial ou fédéral. 


\section{aussi, assez justement, pensons-nous, le style de carrières qui leur était propre. ${ }^{51}$}

51 Sir Charles Boucher de Boucherville illustre un premier type d'appartenance à cette classe politique. Descendant de l'ancien lieutenantgouverneur de Trois-Rivières, son père Pierre-Amable Boucher était seigneur de Boucherville et conseiller législatif. Sa mère, une Sabrevoix de Bleury, était la sœur de C.-C.-S. de Bleury, conseiller législatif. Après des études en Europe, en médecine, il épouse en premières noces la fille d'un des plus grands avocats de Montréal, Suzanne Morrough, et en deuxièmes noces, C. Lussier, fille du seigneur de Varennes. Député sous l'Union, il est nommé président du Conseil législatif à la Confédération et devient ainsi membre du Conseil des ministres. Premier ministre pour la première fois en 1874, à l'âge de 52 ans, il le sera de nouveau en 1891, mais entre ces deux dates il aura eu le temps de démissionner après ses démêlés avec le lieutenant-gouverneur de l'époque, Luc Letellier de Saint-Just, et de devenir tout aussitôt sénateur à Ottawa pour revenir ensuite au provincial en qualité de premier ministre. Il se retirera finalement de la politique pour s'occuper de ses affaires. De 1880-1891, son neveu Tancrède Boucher de Gros Bois siégera comme député à l'Assemblée législative.

Louis-Alexandre Taschereau, élu pour la première fois en 1900, à l'âge de 33 ans, représente un autre type de cette classe politique. Il appartient à une très ancienne famille de la magistrature et de la politique. Son père, Jean-Thomas Taschereau, était juge de la Cour suprême du Canada; sa mère, Joséphine Caron, était la fille de l'ancien lieutenantgouverneur René-Edouard Caron et un de ses oncles, sir Joseph-Adolphe Caron, fut ministre sous Macdonald. Un autre de ses oncles, sir Charles Fitzpatrick, fit une carrière brillante au provincial et au fédéral, puis dans la magistrature, pour enfin devenir lieutenant-gouverneur de la province. Il était allié aussi à une autre famille qui eut de nombreux représentants dans la magistrature et la politique québécoise et canadienne, la famille Cannon. Enfin par son mariage à Aline Dionne, fille d'un conseiller législatif, Taschereau se liait à une vieille famille conservatrice. Pendant qu'Alexandre Taschereau dirigeait la province en temps que premier ministre, son fils siégeait comme député et devint par la suite juge en chef de la Cour suprême. Notons qu'il $y$ eut toujours à la Cour suprême depuis 1867 un membre de la famille Taschereau ou un parent proche de cette famille, c'est-è-dire un Fitzpatrick ou un Cannon.

Honoré Mercier représente enfin un dernier type. Fils de cultivateur, il fera une carrière d'avocat brillante et une carrière politique fulgurante. Comme avocat, il fut entre autres l'associé de Taillon et de Lomer Gouin, qui tous deux deviendront aussi premier ministre. Nul mieux que lui n'incarnera cette nouvelle noblesse, "la noblesse professionnelle née du peuple et qui a succédé à la noblesse titrée" selon le mot de P.-J.-O. Chauveau dans son Charles Guérin. Sa carrière politique et ses affaires le lient à l'élite canadienne-française et canadienne-anglaise du Québec. Son alliance à la famille Gouin, vieille famille bourgeoise, ajoute du prestige à son nom: sa fille cadette Elise épouse son associé Lomer Gouin qui sera longtemps premier ministre du Québec, puis ministre au fédéral avant de devenir lieutenant-gouverneur du Québec. Un des fils de Lomer Gouin deviendra le sénateur Léon Mercier-Gouin, l'autre Paul Gouin sera un des fondateurs de l'A.ction libérale nationale en 1934. Une autre des filles de Mercier épousera Gaspard Fauteux, père de Gaspard Fauteux, lieutenantgouverneur et de Gérald Fauteux, juge de la Cour suprême. Le fils de 
Prenant naissance à la fin du XVIIIème siècle, se développant au cours du XIXème siècle pour arriver au pouvoir dans les années 1850 et constituer une nouvelle classe dirigeante, cette classe politique semble être peu à peu disparue des institutions politiques du Québec à partir de 1920. C'est à ce moment-là que les représentants de la classe moyenne-supérieure entrent de plus en plus nombreux au Conseil des ministres, suivis de quelques représentants des autres classes. La nouvelle élite politique, c'est-à-dire ceux qui détiennent les postes importants au Conseil des ministres, s'alimentera maintenant à la classe moyenne-supérieure, constituée en très grande partie d'individus nés dans les régions rurales et venus s'installer, après des études universitaires qui ont fait d'eux des avocats et des médecins ou encore des membres d'autres professions libérales, dans les deux principales villes du Québec et plus souvent dans les petites villes de province. Ceux qui constituent les figures dominantes de cette nouvelle élite politique viennent généralement à la politique assez jeunes moins de 40 ans, surtout s'ils sont avocats, et généralement font toute leur carrière au niveau provincial ou au niveau fédéral. Il s'agit de quelqu'un qui, fortement ancré dans son milieu (depuis 1919 on a eu tendance à choisir des candidats nés et résidant dans le comté ou du moins y résidant depuis longtemps), fait de sa participation aux institutions publiques une carrière, un métier, qui dépasse en importance celui qui tout d'abord a pu lui servir de tremplin. Contrairement à ce qui était le cas autrefois, ils ne sont pas généralement les plus grands représentants de leur profession, ce qui ne veut pas dire qu'ils ne pouvaient pas le devenir. C'est que la politique les prend très tôt et les accapare presque totalement. Il leur semble beaucoup plus difficile et moins intéressant, sans que ce soit impossible, de poursuivre les deux carrières en même temps.

Moins que la naissance, ce qui constitue la ressource principale de ces nouveaux hornmes politiques c'est une certaine aptitude à monter dans les échelons d'un parti, à se mouvoir

Mercier fut un des principaux ministres de Taschereau et son petit-fils siégera quelque temps comme député à l'Assemblée législative. 
avec facilité et autorité au sein de son organisation et à participer à son contrôle et à son utilisation pour exploiter le pouvoir. Avant tout, il faut être un homme à la fois populaire dans son parti et auprès de l'électorat. Bien sûr, naître dans une famille où le père est ou a été député ou organisateur ne constitue pas un handicap, pas plus d'ailleurs que d'avoir comme beau-père un ancien député ou un ministre ou encore un conseiller législatif. Il est certain que cela permet d'entrer plus vite et plus tôt dans l'intimité des partis et de leur organisation et facilite ainsi la carrière. Cependant, bien que certains individus liés à l'ancienne classe politique dominante participent encore avec succès à la vie politique du Québec, ${ }^{52}$ les députés qui eurent un père ou un beau-père mêlé à la politique et purent en tirer bénéfice comme ce fut le fait de plusieurs députés qui devinrent ministres tels Godbout, Bertrand, Gabias, Cliche, n'appartenaient pas en général à cette classe politique traditionnelle mais plutôt aux classes moyennes qui, à partir de 1920, avaient eu accès aux institutions représentatives et avaient constitué les premiers représentants de cette nouvelle élite politique. S'il demeure encore une certaine différence sociale entre les ministres et les simples députés, elle n'a pas la même signification qu'elle pouvait avoir du temps de la domination de l'ancienne classe politique. Avec celle-ci, c'était une classe socialement dominante qui occupait le pouvoir politique par suite même du contrôle qu'elle détenait sur cette société. Avec l'élite politique nouvelle, il s'agit de dirigeants d'un appareil politique qui ne sont d'aucune manière des détenteurs d'un pouvoir social ou économique dominant, mais d'hommes qui choisissent comme métier de s'emparer du pouvoir politique et de l'exploiter dans l'intérêt général dans la mesure où celui-ci correspond à l'intérêt du parti sans parler de leur intérêt personnel. Ce n'est plus l'aristocratie au pouvoir mais la "partitocratie".

52 Comme $P_{\text {. }}$ Gasgrain, Honoré Mercier fils, ou encore Paul GérinLajoie. Maurice L.-Duplessis en est un autre exemple. Fils de l'honorable Nérée Le Noblet-Duplessis, il était aussi le neveu de Stanislas Cooke, député de Trois-Rivières en 1900, de William Pierre Grant, député libéral de Champlain en 1925, 1927, 1931 à l'Assemblée législative et enfin petitneveu de Charles-Borromée Genest, député de Trois-Rivières en 1869. 
Comment expliquer cet éloignement de la scène politique de la haute-bourgeoisie canadienne-française, pourtant d'origine politique, et son remplacement par des professionnels de la politique dont la ressource première est d'appartenir à un parti et non pas à une classe sociale dominante? Il est difficile de répondre, car si nous constatons sa disparition au sein des institutions parlementaires, sauf la présence de quelques représentants aux Chambres hautes fédérale et provinciale, nous savons très peu de choses sur sa participation à d'autres catégories dirigeantes. Il est possible qu'après s'être développée grâce à la politique et avoir affirmé et confirmé sa position par le contrôle de la politique, cette classe ait émigré vers d'autres élites, professionnelles ou économiques, et que les conseils d'administration aient remplacé les Conseils des ministres. Elle retrouverait ainsi par la participation au contrôle économique un pouvoir d'influence sur la société québécoise, pouvoir qui lui échappait au niveau politique ou qui plus simplement présentait moins d'attrait.

L'ancienne classe politique dominante a pu être poussée dans ce mouvement sous l'influence d'un certain nombre de facteurs. C'est ainsi que les transformations survenues au sein de la collectivité canadienne-française, principalement l'urbanisation, ont peu à peu substitué au système de valeurs hérité de l'ancienne société rurale hiérarchisée un système plus en accord avec une société industrielle, mouvante et toujours plus diversifiée et rendu ainsi plus difficiles certains contrôles sociaux traditionnels. Il faut compter aussi, comme facteur, le développement de la responsabilité étatique aux deux niveaux de gouvernement, développement qui a rendu beaucoup plus contraignante la charge de ministre ou de simple député, par l'allongement des sessions notamment, et rendu ainsi de plus en plus illusoire la possibilité de mener de front deux carrières, l'une professionnelle, l'autre politique. Ce qu'un ancien ministre-avocat nous a déjà déclaré au moment d'une interview est devenu inconcevable: "Je n'ai jamais eu à remettre une cause pour une séance du Conseil." Il y a enfin la place prise par les partis depuis Taschereau et la modification de style qu'ils ont introduite dans notre vie po- 
litique. Ce sont des partis fortement dominés par un chef, des partis relativement stables dans leur "leadership", des partis qui très souvent créent les hommes qui en sont membres ou du moins leur imposent un comportement par une discipline très forte. Si dans son comté le député est devenu plus que jamais un baron, il l'est par le parti et le rôle d'intermédiaire qu'il joue entre les électeurs-clients et le parti et non plus, comme c'était le cas la plupart du temps autrefois, parce qu'il est par la naissance un "seigneur local", ce qui n'implique pas qu'au niveau des électeurs des valeurs anciennes ne continuent pas d'influencer les comportements. La transformation des partis, la place primordiale qu'ils ont prise dans notre vie politique et dans notre vie sociale auraient eu ainsi pour effet de modifier la nature sociale du personnel politique et de faciliter l'implantation dans nos institutions d'un type nouveau d'homme politique. Les difficultés qu'éprouve le parti libéral du Québec à se transformer, les déchirements qui sont aujourd'hui les siens, montrent jusqu'à quel point ce type d'homme s'était enraciné. Ce que Falardeau dit dans son essai sur les élites traditionnelles et les élites nouvelles du Québec pour la période 1940-1960 résume à merveille ce à quoi nous faisons allusion ici :

Le pouvoir politique est autocratique. Il personnalise et surpolitise à la fois ses contacts avec le peuple-électeur en institutionnalisant ses faveurs arbitraires par l'intermédiaire du patronage. Ce sera l'ère du député-entrepreneur-homme d'affairescommanditaire-distributeur de largesses: l'EtatProvidence à l'heure des anciens clochers québécois. ${ }^{53}$

Les hommes politiques $d u$ Québec: élite dirigeante ou simplement dominante?

C'est donc dans les mains de cette bourgeoisie développée depuis la fin du XVIIIème siècle par la politique, la magistrature et quelquefois les affaires, qu'échoit ce nouveau pouvoir en 1867. Grâce à lui elle va devenir encore plus importante et verra s'accentuer encore davantage son caractère

53 J.-C. Falardeau, op. cit., 138. 
d'élite. Elle formera à l'intérieur de la société canadiennefrançaise une "upper class" (classe supérieure) épousant par beaucoup de côtés les caractères d'une aristocratie. Modelant, grâce au contrôle qu'elle exerce sur le pouvoir politique provincial, la société canadienne-française, sa pensée et sa vie, elle sera aussi au niveau fédéral l'interprète de ses aspirations et cela avec d'autant plus de facilité qu'on lui abandonne cet électorat, ${ }^{54}$ et que par la magistrature elle veille à la conservation des valeurs dont elle s'inspire dans son action politique. Il ne s'agit pas ici d'établir que de sombres complots animaient les divers membres de cette classe politique mais beaucoup plus simplement de rappeler qu'à toute une série de postes-clés, du point de vue du contrôle qu'ils permettaient sur la société québécoise, étaient présents les membres d'une même classe sociale, partageant dans leur ensemble les mêmes valeurs et les mêmes intérêts, ce qui n'exclut pas la possibilité de conflits.

Cette classe politique, par l'intermédiaire d'hommes comme Taschereau et Duplessis, verra son influence prolongée, alors même qu'un personnel politique socialement différent lui aura succédé au sein des institutions politiques. Ces hommes nouveaux qui constituent après 1920 la nouvelle élite politique proviennent surtout de la classe moyenne-supérieure, mais aussi des classes moyenne-inférieure, paysanne et ouvrière. Toutefois les représentants de ces dernières classes s'intégreront à la classe moyenne-supérieure tout comme les représentants de celleci s'étaient très souvent intégrés à la classe politique traditionnelle au XIXème siècle. Le changement d'élite politique marquera le style politique du Québec, il ne modifiera que très peu l'orien ${ }^{+}$ation de la pensée sociale et économique des gouvernants. Il faudra attendre les années cinquante pour qu'une contestation apparaisse, une contestation débouchant sur de nouveaux rapports sociaux et économiques de même que sur de nouveaux rapports entre le peuple et ses gouvernants et sur le rôle de l'État (voir notamment la revue Cité Libre).

Il y aurait eu ainsi succession au pouvoir d'une élite politique à une autre sans que pour autant cela ait changé d'une

64 Pierre Elliott Trudeau, op. cit. 
manière marquante l'orientation générale donnée à la société canadienne-française. Pourquoi? Là réside finalement la question fondamentale. Selon nous, les faits suivants expliqueraient en très grande partie ce phénomène: si "grâce" à 1867 les Canadiens français recoivent au niveau provincial le contrôle d'un pouvoir politique et au niveau fédéral la participation à un autre pouvoir politique, ils ne pourront agir qu'à l'intérieur d'un cadre social et économique leur imposant des limites d'action étroites, limites que seule une vie démocratique intense et une conscience collective et nationale vive et réaliste auraient pu repousser plus loin, sinon briser. C'est ce qui fut impossible pendant longtemps et demeure difficile encore maintenant, parce que ces hommes politiques, qu'ils aient appartenu à une classe sociale dominante ou à une élite partisane, ne pouvaient exercer ce pouvoir et le conserver tranquillement qu'en acceptant d'être dans une situation de dépendance face aux autres catégories dirigeantes ${ }^{55}$.

$\mathrm{Au}$ moment où les Canadiens français entrent en possession d'un pouvoir politique nouveau, des bouleversements considérables ont déjà commencé de se produire au Québec, mais aussi au Canada, sur le plan économique et social sous l'effet de la révolution industrielle. Ce qu'il faut rappeler ici, du point de vue qui nous occupe, c'est que, qu'il s'agisse de la phase précédant la révolution industrielle et pendant laquelle l'exploitation de la forêt a pris une dimension considérable, ou de l'industrialisation basée dans sa première phase sur l'industrie manufacturière et dans sa deuxième phase sur l'exploitation des richesses naturelles, tout au long de cette très longue période qui commence avant la Confédération et l'accompagne ensuite jusqu'à aujourd'hui, les liens entre pouvoir économique et pouvoir politique furent très étroits, comme dans la plupart des pays, mais aussi selon des modalités particulières. Le développement économique s'est fait à même l'exploitation de richesses naturelles appartenant à l'Etat, de services publics comme les chemins de fer

55 Ce n'est que lorsque nous aurons pu analyser les types de rapports qui ont pu exister: entre l'élite politique et les autres minorités dirigeantes que nous pourrons réellement percevoir la nature du pouvoir qui fut le sien. 
subventionnés par l'État, d'une main-d'œuvre nombreuse et bon marché. Dans un cas comme dans l'autre, on avait besoin de la "compréhension" des détenteurs du pouvoir politique pour pouvoir obtenir, conserver et exploiter, avec le plus grand profit et la plus grande tranquillité possible, ces richesses. Les hommes politiques du Québec pouvaient-ils se permettre de ne pas être conciliants ? La plupart de nos gouvernants avaient été formés à l'école du libéralisme économique, doctrine correspondant très bien d'ailleurs à leur fort désir d'ascension sociale individuelle. De plus, des liens étroits existaient souvent, soit par la parenté ou par les affaires - plusieurs membres de l'ancienne classe politique étaient les avocats des grandes compagnies - soit plus simplement par intérêt personnel (participation à des conseils d'administration) ou partisan (subventions à la caisse électorale). La transformation de la société québécoise et l'ampleur prise par le phénomène partisan rendirent encore plus couteuse la conservation du pouvoir, cependant que l'industrialisation accélérée du Québec à partir de 1939 multiplia les occasions de conflits et de compromis entre hommes politiques et hommes d'affaires.

Encore faut-il rappeler deux autres caractères des relations du pouvoir économique et du pouvoir politique au Québec; il s'agit d'un pouvoir économique de caractère international prenant appui sur des grandes entreprises et des puissances financières considérables, souvent mieux organisées et renseignées que le pouvoir politique, ${ }^{56}$ représentant très souvent une puissance financière dépassant de beaucoup les petits budgets provinciaux; deuxièmement, son caractère étranger, les détenteurs de ce pouvoir étant massivement des Anglais, des Canadiens anglais et de plus en plus des Américains.

Ainsi, sur le plan de l'économie, il semble que les rapports entre pouvoir économique et pouvoir politique aient été loin d'être égaux. Vulnérable, le Québec l'était dans ses structures

56 Albert Faucher, "Pouvoir politique et pouvoir économique dans l'évolution du Canada français", dans Le Pouvoir dans la Société canadiennefrançaise, 61-79. 
administratives, dans sa puissance financière, mais aussi dans ses hommes politiques qui non seulement étaient la plupart du temps en accord avec ce type de relation mais encore tiraient profit de cette dépendance. Ces hommes politiques pouvaient difficilement être autre chose que des intermédiaires, ils furent des intermédiaires satisfaits. Ils purent donner l'impression d'être entrés en possession d'une forteresse politique québécoise grâce à la Confédération, peut-être même le croire: cette forteresse était en fait non seulement assiégée mais investie. Ils étaient les gouverneurs d'une place forte tombée sous le contrôle de forces économiques extérieures à cette société.

Limités sur le plan économique par suite des rapports existant entre un pouvoir politique canadien-français et un pouvoir économique ethniquement différent, ils le furent aussi sur le plan social et culturel. Et cette fois, il s'agit de rapports qui existèrent entre le pouvoir politique et des pouvoirs sociaux intérieurs à la société canadienne-française et principalement le clergé.

La société dans laquelle s'inséra le nouveau système politique du Québec en 1867 était une société fortement structurée socialement et intégrée culturellement. S'il est vrai que par suite de l'industrialisation et de l'urbanisation les structures sociales allaient connaître une profonde mutation, certains pouvoirs sociaux et le système de valeurs qu'ils avaient développé et qui leur servait de légitimation, notamment la valeur nationale, déjà fortement implantés allaient continuer d'encadrer puissamment cette société. Les structures de base de l'ancienne société rurale, familles, paroisses, pourront être complètement bouleversées par la révolution industrielle, de nouvelles activités apparaître, la population active se diversifier, les élites dirigeantes préserveront leur pouvoir et continueront jusqu'à une époque récente de présenter du Québec et au Québec la même image de lui-même. ${ }^{5 r}$

Tout au cours du XIXème siècle, accompagnant l'affaiblissement de la petite aristocratie foncière, deux groupes ont déve-

57 Fernand Dumont, op. cit. 
loppé leur emprise sur la société québécoise, la nouvelle bourgeoisie des professions libérales et le clergé. Les deux élites, après une première période d'opposition, de 1820 à 1860 environ, deviendront, côte à côte, les définisseurs de situation de cette société, des définisseurs plus souvent d'accord qu'en conflit. Après avoir été divisés entre une tendance radicale et une tendance ultramontaine concernant les fins à poursuivre et la nature du pouvoir, les membres de cette bourgeoisie seront par la suite réunis, unifiés, les deux tendances devenant les ailes extrémistes et de moins en moins influentes des deux partis politiques, idéologiquement peu différents. Administrative, politique, industrielle, religieuse, intellectuelle, cette élite bourgeoise sera majoritairement d'accord sur un certain nombre de valeurs, de mythes plutôt que d'objectifs, de thèmes qui constitueront ce que l'on a appelé par la suite l'idéologie unitaire, ${ }^{58}$ idéologie qui consacrait l'entente cordiale entre la bourgeoisie et les différents pouvoirs qu'elle occupait et le clergé. Cette entente dura jusqu'à tout récemment, parce que les premiers détenteurs du pouvoir politique, la classe politique dominante de la fin du XIXème siècle, avaient été, avec le clergé, les créateurs de cette idéologie et les premiers à pouvoir la diffuser, et parce que leurs successeurs, formés très majoritairement dans les institutions de diffusion par excellence de cette idéologie, les séminaires et collèges classiques, trouvaient dans son respect ou son exploitation un profit politique certain et une tranquillité sociale rassurante.

Ainsi par son origine sociale et les limites étroites à l'intérieur desquelles elle eut à agir, limites économiques, sociales, constitutionnelles, ${ }^{69}$ l'élite politique canadienne-française pouvait très difficilement ne pas être conservatrice et condamnée à jouer un rôle d'intermédiaire auprès des pouvoirs sociaux et économiques dominants. Elle fut l'intermédiaire nécessaire, sou-

58 Guy Rocher et Fernand Dumont, "Introduction à une sociologie du Canada français", Recherches et Débats du Centre catholique des intellectuels français, cahier no 34 (Arthème Fayard, mars 1961): 13-38.

59 C'est volontairement que nous avons ignoré les limites d'ordre constitutionnel, conscient qu'il s'agissait là d'un point abondamment discuté ailleurs. Le lecteur aurait tort de croire cependant que nous les jugeons peu importantes. 
vent contraint, souvent satisfait, à la gouverne d'une société colonisée. La seule ressource, bien à elle, qu'elle aurait pu utiliser pour repousser plus loin ces limites, pour y faire véritablement face, aurait été de susciter, d'entretenir une vie démocratique au Québec, afin de trouver - dans une société consciente de son âme et des conditions de son avenir - l'appui indispensable pour un usage réel du pouvoir politique tombé entre leurs mains. Des traditions déjà implantées depuis longtemps dans notre culture politique avaient faussé le sens véritable des institutions et des procédures de la démocratie parlementaire et empêché cette conscience collective; des intérêts de classe, de partis, de groupes ethniques, de pouvoirs économiques ou sociaux avaient produit une atomisation de la société canadienne-française, une atomisation facilitée, cachée par l'appel constant fait à une idéologie nationaliste limitée à des usages électoraux. Atomisée, la société canadienne-française a eu presque toujours une élite politique de type colonial.

Seule une prise de conscience collective peut redonner à cette société le sens de son identité, de ses possibilités, de ses besoins et des exigences qu'ils entraînent. Seule une vie démocratique peut permettre la survie de cette conscience et le contrôle par le peuple des individus chargés de mesurer constamment la marge entre les aspirations et les possibilités et de prendre les décisions d'orientation. La seule arme que la société canadienne-française ne possède réellement pas est celle que possède tout groupe social défavorisé, économiquement démuni, comme c'est le fait de la classe ouvrière, la force d'une masse consciente. Dans ce sens nous serions presque tenté de dire que les Canadiens français sont "condamnés à la démocratie et que tout autre mode de vie ne peut que leur être fatal". C'est la signification profonde et l'enjeu de ce que l'on a appelé la Révolution tranquille.

ROBERT BOILY 